\title{
Enhanced cholinergic suppression of previously strengthened synapses enables the formation of self-organized representations in olfactory cortex
}

\author{
Christiane Linster, ${ }^{*}$ Michaella Maloney, Madhvi Patil, and Michael E. Hasselmo* \\ Department of Psychology Center for Memory and Brain and Program in Neuroscience, Boston University, 2 Cummington Street, \\ Boston, MA 02215, USA
}

Received 4 June 2003; revised 17 July 2003; accepted 22 July 2003

\begin{abstract}
Computational modeling assists in analyzing the specific functional role of the cellular effects of acetylcholine within cortical structures. In particular, acetylcholine may regulate the dynamics of encoding and retrieval of information by regulating the magnitude of synaptic transmission at excitatory recurrent connections. Many abstract models of associative memory function ignore the influence of changes in synaptic strength during the storage process and apply the effect of these changes only during a socalled recall-phase. Efforts to ensure stable activity with more realistic, continuous updating of the synaptic strength during the storage process have shown that the memory capacity of a realistic cortical network can be greatly enhanced if cholinergic modulation blocks transmission at synaptic connections of the association fibers during the learning process. We here present experimental data from an olfactory cortex brain slice preparation showing that previously potentiated fibers show significantly greater suppression (presynaptic inhibition) by the cholinergic agonist carbachol than unpotentiated fibers. We conclude that low suppression of non-potentiated fibers during the learning process ensures the formation of self-organized representations in the neural network while the higher suppression of previously potentiated fibers minimizes interference between overlapping patterns. We show in a computational model of olfactory cortex, that, together, these two phenomena reduce the overlap between patterns that are stored within the same neural network structure. These results further demonstrate the contribution of acetylcholine to mechanisms of cortical plasticity. The results are consistent with the extensive evidence supporting a role for acetylcholine in encoding of new memories and enhancement of response to salient sensory stimuli.
\end{abstract}

(C) 2003 Published by Elsevier Inc.

\section{Introduction}

The specific cellular effects of acetylcholine play an important role in setting the dynamics of cortical structures. Blockade of these cellular effects with muscarinic acetylcholine receptor antagonists such as scopolamine and atropine causes strong behavioral impairments. At high doses, these drugs cause delirium and hallucinations - a profound breakdown in cognitive processes (see Kopelman, 1986 for review). At lower

\footnotetext{
${ }^{*}$ Corresponding authors. Fax: +617-353-1424 (M.E. Hasselmo), Present address: Department of Neurobiology and Behavior, W245 G. Seeley Mudd Hall, Ithaca, NY 14853. Fax: +607-2544308 (C. Linster).

E-mail addresses: CL243@cornell.edu (C. Linster), hasselmo@bu.edu (M.E. Hasselmo).
}

doses, these antagonists cause impairments in specific cognitive tasks, including encoding of verbal stimuli for subsequent retrieval (Atri et al., 2003; Ghonheim \& Mewaldt, 1977), encoding of visual stimuli for subsequent recognition (Aigner \& Mishkin, 1986; Sherman, Atri, Hasselmo, Stern, \& Howard, 2003) and response to stimuli in a continuous performance tasks (Wesnes and Warburton, 1983). Selective lesions of cholinergic innervation cause decreases in perceptual discriminability (Linster, Garcia, Hasselmo, \& Baxter, 2001) and impairments in continuous performance tasks (McGaughy, Kaiser, \& Sarter, 1996; McGaughy \& Sarter, 1998). The research presented here focuses on the olfactory system. In the olfactory system, scopolamine has been shown to reduce behavioral odor habituation (Hunter \& Murray, 1989), impair short-term memory 
for odors (Ravel, Elaagouby, \& Gervais, 1994), and cause increased interference between overlapping odors (DeRosa \& Hasselmo, 2000). Scopolamine also prevents increases in odor discrimination in a conditioning task (Fletcher \& Wilson, 2002), while causing greater crosshabituation in olfactory cortex activity (Wilson, 2001).

These different effects can be linked to the loss of cellular effects of acetylcholine common to most cortical structures, including piriform cortex, hippocampus and neocortex (see review in Hasselmo \& McGaughy, 2003). Acetylcholine causes similar physiological effects in most cortical structures, including direct depolarization of neurons (Barkai \& Hasselmo, 1994; Benardo \& Prince, 1982; Madison \& Nicoll, 1984) and enhancement of long-term potentiation (Blitzer, Gil, \& Landau, 1990; Brocher, Artola, \& Singer, 1992; Patil, Linster, Lubenov, \& Hasselmo, 1998). Experimental data in a number of different regions also shows that acetylcholine strongly suppresses excitatory glutamatergic synaptic transmission via presynaptic inhibition at intrinsic, recurrent synapses in cortical structures (Dutar \& Nicoll, 1988; Gil, Connors, \& Amitai, 1997; Hasselmo \& Bower, 1992; Hasselmo, Schnell, \& Barkai, 1995; Hounsgaard, 1978; Hsieh, Cruikshank, \& Metherate, 2000; Linster, Wyble, \& Hasselmo, 1999; Williams \& Constanti, 1988). In contrast, acetylcholine usually causes less presynaptic inhibition at afferent fiber synapses (Gil et al., 1997; Hasselmo \& Bower, 1992; Hasselmo et al., 1995; Hsieh et al., 2000; Linster et al., 1999). The research presented here focuses on the functional role of this cholinergic presynaptic inhibition of excitatory glutamatergic synaptic transmission.

Because neuromodulators such as acetylcholine have such diffuse effects, the use of computational models proves very important for understanding the role of acetylcholine in changing the functional dynamics of cortical regions. In particular, a growing number of computational models have addressed the associative memory function of neural structures such as the olfactory cortex or hippocampus. Most of these modeling efforts have relied on the idea that the broadly distributed intrinsic fibers in these cortical networks strongly resemble a class of abstract models called associative memories (Anderson, 1972; Haberly, 1985; Haberly \& Bower, 1989; Hasselmo, Anderson, \& Bower, 1992; Hasselmo \& Bower, 1993; Hasselmo et al., 1995; Kohonen, 1984; Marr, 1971; McNaughton \& Morris, 1987; Wilson \& Bower, 1992). These intrinsic connections give models of the olfactory cortex and the hippocampus the capacity to store distributed patterns (conveyed via afferent input connections) and to subsequently perform pattern completion on degraded or noisy versions of these input patterns. Simulations of associative memory function in these cortical networks have led researchers to investigate how physiologically realistic neural networks could store extensively overlapping patterns, as would be expected for natural stimuli. It has been shown that memory capacity can be enhanced through minimization of interference between overlapping patterns during the learning process; this minimization can be obtained by selective cholinergic suppression of excitatory synaptic transmission at intrinsic, but not afferent, connections (Hasselmo \& Bower, 1992; Hasselmo et al., 1992, 1995).

Computational modeling has demonstrated that memory capacity can also be enhanced by recruitment of additional neurons, which are not directly activated by afferent input, through a self-organizing process (Carpenter \& Grossberg, 1993; Levy, 1996; Wallenstein \& Hasselmo, 1997; Wu, Baxter, \& Levy, 1996). In this paper, self-organization refers to models in which the pattern of activity is not directly imposed by external input. Instead, self-organization models contain populations of neurons which gradually form new patterns of response during presentation of a series of sensory stimuli, until the network reliably shows a distinct pattern of response to each input stimulus (Ambros-Ingerson, Granger, \& Lynch, 1990; Carpenter \& Grossberg, 1993; Grossberg, 1976; Hasselmo, 1995). This gradual self-organization results from modification of afferent input synapses which are the primary influence on postsynaptic activity during encoding. In contrast, associative memory models focus on storage of associations between patterns of activity imposed by external input. Thus, the input arrives along afferent input connections which have been previously modified and do not change further, while associations are encoded via strengthening of excitatory recurrent feedback synapses between neurons activated by the external input (Anderson, 1972; Haberly, 1985; Haberly \& Bower, 1989; Hasselmo \& Bower, 1993; Kohonen, 1984). Because the fibers in associative memory models are storing associations without changing the pattern of response to a complete external stimulus, this involves strengthening of synapses which are not the primary influence on post-synaptic activity of neurons (Hasselmo, 1995).

At first sight, these two paradigms seem mutually exclusive, since the suppression of intrinsic excitatory connections would impair the formation of self-organizing representations which depend upon recruitment of neurons that are not directly activated by afferent input during the learning process. In this paper we show that, in a network model of olfactory cortex, self-organized representations can be formed even in the presence of cholinergic suppression of excitatory synaptic transmission, if synapses that have been potentiated during the learning process are more highly suppressed than unpotentiated synapses. We also show that in a brain slice preparation of olfactory cortex, previously potentiated fibers are indeed proportionally more suppressed than unpotentiated fibers. The inclusion of the suppression parameters obtained from the brain slice 
experiment into the model of olfactory cortex allows self-organization in the model to reduce the overlap between highly overlapping patterns through the recruitment of additional (context) neurons by each pattern during learning.

\section{Materials and methods}

\subsection{Computational modeling}

The computational model presented here allows analysis of odor storage in piriform cortex through formation of associations between different components of an odor in anterior piriform cortex, and self-organization of additional odor responses in the posterior piriform cortex. The model draws on extensive previous anatomic and physiological research on the olfactory cortex (Haberly, 1985; Haberly \& Bower, 1989).The model consisted of 40 pyramidal cells in both the anterior and the posterior portion of the network, and 40 each of feedback and feedforward interneurons. Afferent input was given to anterior pyramidal cells and feedforward interneurons. Each pyramidal cell made recurrent excitatory connections to other pyramidal cells with a relatively low probability $(12 \%$ between anterior neurons, $12 \%$ between anterior and posterior neurons, and $12 \%$ between posterior neurons) and initial synaptic weights drawn from a uniform random distribution between 0.225 and 0.275 . These connections were made via synapses using both fast time courses (AMPA) and slow time courses (NMDA). Each feedforward interneuron connected to 5 anterior pyramidal cells (with a synaptic strength of 0.2 ). These connections were inhibitory and had fast time courses representing GABAA receptors. Pyramidal cells and feedback interneurons were reciprocally connected with $20 \%$ probability via fast synapses (AMPA resp. GABA-A). Afferent input from the lateral olfactory tract was simulated as $120 \mathrm{~ms}$ bursts of activity to anterior pyramidal cells and feedforward interneurons. In the simulations described here, $20 \%$ of anterior pyramidal cells and feedforward neurons were activated by a given input pattern. The equations used to calculate neural and synaptic activities (conductance based probabilistic integrate and fire neurons), as well as the parameter values used for neural and synaptic time constants and reversal potentials were those used previously (Hasselmo, Linster, Patil, Ma, \& Cekic, 1997).

Each synaptic weight $w$ between two pyramidal cells was composed of its initial weight $w_{\text {naive }}$ at the beginning of the simulation and its potentiated component $w_{\text {pot }}$, (that is $w=w_{\text {naive }}+w_{\text {pot }}$ ). At the beginning of the simulations, $w_{\text {naïve }}$ was given a small random value and $w_{\text {pot }}$ was set to zero. During the learning process $w_{\text {pot }}$ was changed according to a simple Hebbian learning rule by which $w_{\text {pot }}$ was incremented by $\eta(\eta=0.2)$ when a presynaptic and a postsynaptic action potential happened coincidentally in a time window of $4 \mathrm{~ms}$. Presynaptic normalization was applied at each time step which kept the sum $\sigma$ of all synaptic weights connecting a given neuron to other neurons in the network constant $(\sigma=2.0)$. Cholinergic suppression during the learning process was implemented by multiplying each of the components of the synaptic weights by a suppression factor $\alpha$ so that the total synaptic weight $w$ was lower during learning:

$w=w_{\text {nä̈ve }}\left(1-\alpha_{\text {naïve }}\right)+w_{\text {pot }}\left(1-\alpha_{\text {pot }}\right)$.

This enabled us to test the effect of differential suppression of the initial and potentiated components of the synaptic weights. For comparisons between different combinations of suppression parameters during learning, we compared the overlap between neural response patterns elicited by two stored patterns after the learning process. We ran 25 simulations for each combination of suppression parameters, varying both $\alpha_{\text {naive }}$ (the suppression of initial synaptic strength) and $\alpha_{\text {pot }}$ (the suppression of newly potentiated synaptic strength). For each trial run, a new network with randomized initial conditions was built, and two random input patterns with $50 \%$ overlap between them were chosen. Each input pattern activated 8 out of 40 anterior pyramidal cells and 8 out of 40 feedforward interneurons. During the learning process, each pattern was presented to the network once during $120 \mathrm{~ms}$ and the synaptic weights between pyramidal cells were updated according to the Hebbian learning rule described above. The calculated synaptic changes became effective immediately and could thus influence the subsequent learning process. After the learning process, the synaptic weights were kept constant and the neural responses to the stored patterns were compared by calculating the normalized dot products between the neural activities elicited by each of the patterns (Hasselmo et al., 1992).

Electrophysiology. Electrophysiological work in brain slice preparations of the piriform cortex allowed testing of whether cholinergic modulation had a stronger effect on synapses which had previously been strengthened with repetitive stimulation. Slices of piriform cortex were prepared from adult female albino Sprague-Dawley rats (150-200 g; 6-10 weeks of age) following standard procedures (Hasselmo \& Bower, 1992; Hasselmo et al., 1997; Patil and Hasselmo, 1998; Patil et al., 1998) and in accordance with institutional guidelines. Extracellular recordings were taken using glass-pipettes of about $5 \mathrm{M} \Omega$ impedance and filled with $3 \mathrm{M} \mathrm{NaCl}$. The orthodromic stimuli were delivered through a NeuroData PG4000 stimulator, using fine unipolar tungsten electrodes. The stimulation and recording electrodes were placed in layer $\mathrm{Ib}$ under direct visual guidance. Experiments were only analyzed if they displayed stable 
responses; those with any indication of epileptiform activity were discarded. In addition, experiments were excluded from the analysis if the synapse did not recover to the after-LTP baseline level (ACSF baseline level for the controls) following the period of cholinergic suppression by carbachol. The response to maximal test pulse stimulation in recovery had to be of a magnitude similar to the corresponding responses acquired in the after-LTP or ACSF baseline interval for experimental and control protocols, respectively. Slices that were degrading during the experiment, or those that had died by the time the testing had concluded were eliminated with this criterion. For analysis, the onset slopes of the population EPSPs were calculated automatically. For comparisons within an experimental condition, paired $t$ tests were applied to the calculated slopes. For comparisons of the cholinergic suppression between the experimental and the control slices, single factor analysis of variance was applied to the percentage values of the suppression calculated from the ratio between the suppressed and the reference potentials.

\section{Results}

3.1. Effect of selective cholinergic suppression of previously potentiated synapses on associative learning and self-organization

The initial research using the computational model demonstrated properties important to the self-organization of odor responses in the posterior piriform cortex. This extended work on previous olfactory cortex models (Barkai, Bergman, Horwitz, \& Hasselmo, 1994; Hasselmo et al., 1992; Hasselmo \& Bower, 1993; Wilson \& Bower, 1992; Wilson, 1988), which focused on the associative memory function of recurrent synapses in anterior piriform cortex. The model presented here includes the additional feature that pyramidal cells are not all driven by direct afferent input from the olfactory bulb. Instead, activity in the posterior part of the model depends upon the spread of activity from more anterior regions, as suggested by the anatomy of the olfactory cortex (Haberly, 1985; Haberly \& Bower, 1989; Haberly \& Price, 1977; Wilson \& Bower, 1992) (Fig. 1A). We have shown that in this framework, the activity in response to an input pattern from the olfactory bulb spreads from anterior to posterior neurons with successive sniff cycles if activity dependent plasticity is allowed on association fibers (Linster, Hasselmo, \& Gervais, 1995). In this model, cholinergic suppression of all intrinsic association fibers during learning, as previously proposed (Barkai et al., 1994; Hasselmo, 1995; Hasselmo \& Bower, 1993; Hasselmo et al., 1995; Hasselmo \& Wyble, 1997), interferes with the spread of activity from anterior to posterior neurons in the model.
This interference with spread of activity slows or blocks the formation of self-organized representations in posterior neurons (Fig. 1C, Strong suppression). However, as shown previously, no suppression during learning leads to high interference between simultaneously stored patterns and to runaway neural activity (Fig. 1C, No suppression). Effective function in the network requires that cholinergic suppression of association fibers be selective for previously modified synapses (Fig. 1C, Selective suppression), thus preventing the interference of previously learned patterns (via high suppression of potentiated fibers), while still allowing the self-organization based on initial conditions (via lower suppression of the non-potentiated fibers). Thus, based on self-organization of odor responses in the posterior piriform cortex, the model predicts that cholinergic suppression of excitatory synaptic potentials should be stronger for synapses strengthened with a long-term potentiation paradigm, while having a weaker effect on the initial strength of synapses which have not been potentiated.

The selective suppression of potentiated synapses during encoding prevents the self-organization from being dominated by potentiated synapses. With no suppression, potentiated synapses dominate the self-organization of new representations, causing the network to respond to each new input with a pattern resembling the response to previous inputs (notice the similarity in the pattern of activity in posterior piriform cortex in the "No Suppression" case in Fig. 1C). Thus, the self-organization lumps all representations together. In contrast, with selective suppression, the self-organization can evenly distribute new representations based on the weak random initial connectivity pattern, rather than the strong potentiated connectivity. With complete suppression of all synapses, the random initial connectivity cannot induce initial responses and self-organization in posterior piriform cortex does not occur. Note that the time course of the sensitivity to suppression should extend beyond just a short period of time. Even representations formed days in advance could dominate self-organization to new inputs. Thus, if there is a loss of sensitivity to suppression, it should occur over an extended period. Suppression becomes less necessary as the representation across neurons becomes more evenly balanced (due to experience with a wide range of inputs) or becomes noisier (possibly due to physiological processes randomizing the strength of synaptic connections).

\subsection{Brain slice experiments to test the prediction of selective cholinergic suppression of previously potentiated synapses}

We tested the prediction that previously potentiated synapses should be more highly suppressed by cholinergic modulation in an in vitro brain slice preparation of 


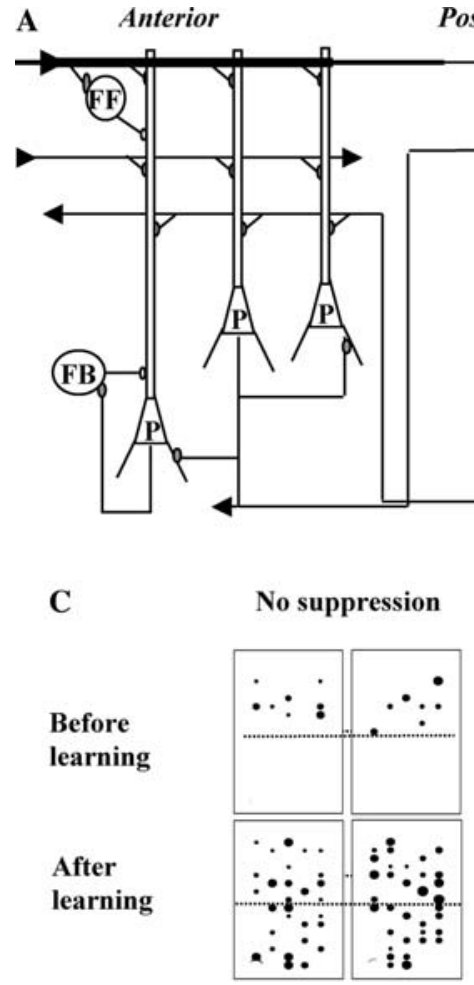

Odor A Odor B
Posterior

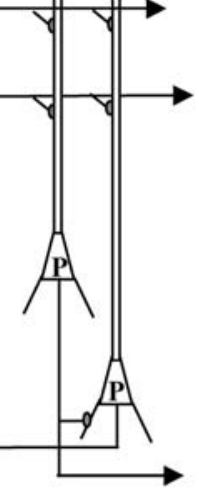

B

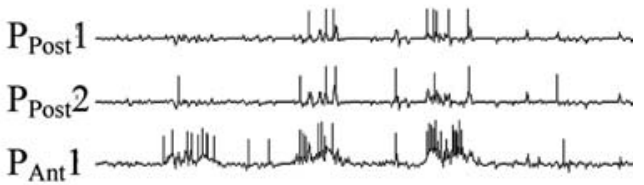

$P_{\text {Ant }}$

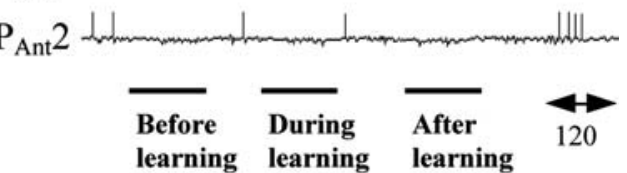

Strong suppression Selective suppression

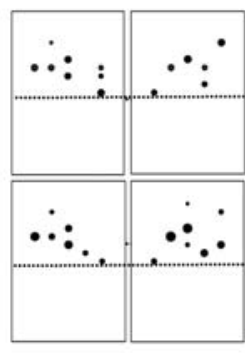

Odor A Odor B

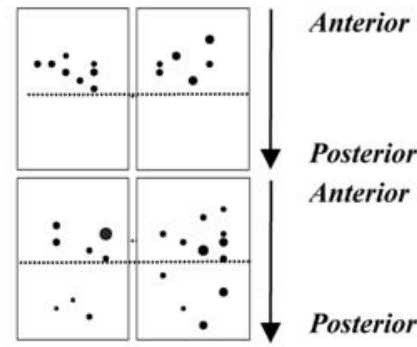

Odor A Odor B

Fig. 1. Schematics and simulations of computational model. (A) The model incorporates pyramidal cells (P), feedforward (FF), and feedback interneurons (FB). Whereas in previous models the probability that a pyramidal cell received afferent input decreased linearly from anterior to posterior pyramidal cells (Hasselmo et al., 1997), in the simulations presented here, all pyramidal cells and feedforward interneurons in the anterior portion of the network receive afferent input, whereas no neurons in the posterior network receive input. This simplification was made to enable the analysis of the contribution of self-organized representations to the representations of patterns in the network. Feedforward interneurons inhibit surrounding pyramidal cells. All pyramidal cells receive excitatory and plastic synapses from all other pyramidal cells with a relatively low probability. Pyramidal cells also exit a small proportion of feedback interneurons which in turn inhibit certain pyramidal cells. All connections are made randomly with constraints detailed in the methods. (B) Membrane potentials and action potentials of two anterior and two posterior pyramidal cells in response to an input pattern before learning, during learning and after learning. Note that posterior neurons do not respond to afferent input before the learning process. (C) Average activity patterns of neural responses in the model before and after learning with three different combinations of synaptic suppression during learning. Average activities (number of action potentials during the application of the stimulus) of all neurons in the simulations are represented by the size of the dots. The network used in these simulations contained 40 anterior and 40 posterior pyramidal cells. In each frame, anterior neurons are located in the top half of the frame and posterior neurons in the bottom half (separated by the dotted line). Each graph shows the average neural activation in response to two different, but highly overlapping ( $50 \%$ overlap) input patterns (Odor A and B) before learning (upper frame) and after learning (lower frame). During learning, each of the input patterns is presented during $120 \mathrm{~ms}$ and the synaptic weights are updated according to a simple Hebbian learning rule (see methods). No suppression: if the synaptic weights are not suppressed during the learning process, the high overlap between the two input patterns leads to high interference and additional neurons are recruited in both anterior and posterior parts of the network. Strong suppression: all synaptic weights (including both the initial and the potentiated components of the synaptic weight) were suppressed by $75 \%$ during the learning. Because of the suppression of the initial component of the synaptic weights, posterior neurons are not activated by the input patterns during the learning process and the connections from anterior to posterior neurons are not strengthened. As a consequence, no posterior neurons respond to the patterns after completion of the learning process. Selective suppression: when only the potentiated, but not the initial components of the synaptic weights are suppressed during the learning process, posterior neurons can become activated while runaway activity is prevented.

piriform cortex, in which selective suppression of intrinsic but not afferent fibers by the cholinergic agonist carbachol (CCh) has been previously analyzed in detail (Hasselmo \& Bower, 1992; Patil \& Hasselmo, 1999). In brain slices of piriform cortex, long term potentiation can be induced at both afferent synapses, consisting of projections from the olfactory bulb via the lateral olfactory tract, and at intrinsic fiber synapses, providing recurrent connections between pyramidal cells within the cortex (Hasselmo \& Barkai, 1995; Jung, Larson, \& Lynch, 1990; Kanter \& Haberly, 1990, 1993; Patil et al., 1998). In order to study the effect of CCh on excitatory synaptic transmission in previously potentiated fibers, we adopted the stimulation paradigm described by Kanter and Haberly (1993) to induce LTP in the intrinsic fiber pathway in vitro (Fig. 2A) and we recorded extracellular population EPSPs in response to electrical stimulation of the intrinsic fiber pathway (Fig. 2B). The 
A

$$
\begin{aligned}
& \text { Stimulating } \\
& \text { electrodes }
\end{aligned}
$$

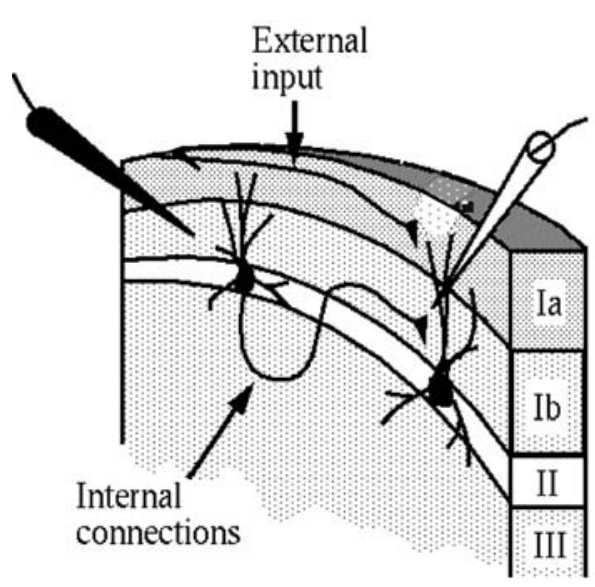

B

$$
50 \mu \mathrm{M} \mathrm{CCh}
$$

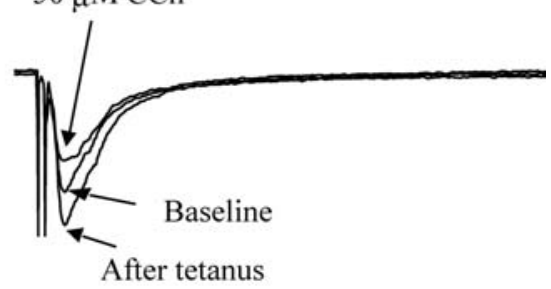

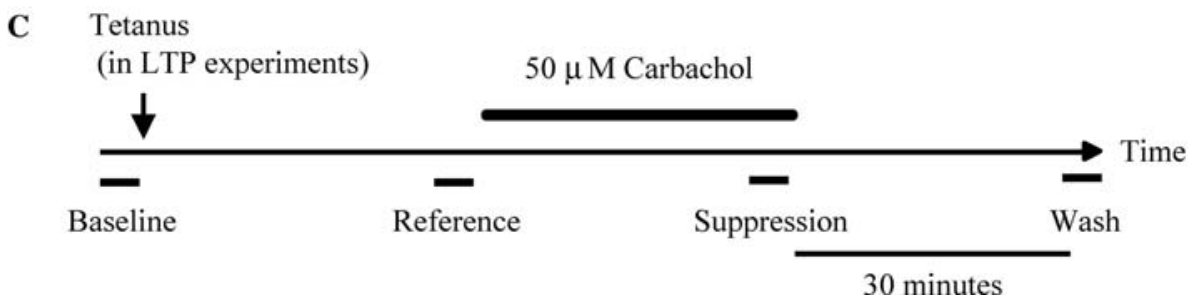

Fig. 2. Experimental methods and protocol. (A) Schematic illustration of the brain slice preparation. Recording and stimulation electrodes were placed in layer $\mathrm{Ib}$ of the piriform cortex under visual guidance and extracellular field potential recordings were obtained. (B) Potentials recorded in response to stimulation of layer Ib (association fibers) in layer Ib. Baseline: Baseline established during first 5 min of recording; After tetanus: potentials recorded $30 \mathrm{~min}$ after the potentiating train; $50 \mu \mathrm{MCCh}$ : potential recorded $30 \mathrm{~min}$ after the bath application of $50 \mu \mathrm{M} \mathrm{CCh}$. For analysis, the slopes at half-amplitude of the potentials were calculated using custom-written software. (C) Experimental protocol. A baseline potential amplitude was established in ACSF using test pulses at 30s intervals at a shock strength pre-adjusted to 50-60\% of the maximal response (Baseline). Throughout the experiment, test pulses were delivered at $30 \mathrm{~s}$ intervals at the same strength. In experimental slices, a potentiating stimulus train (10 sets of 4 pulses at $100 \mathrm{~Hz}$ at $200 \mathrm{~ms}$ intervals (Kanter \& Haberly, 1993; Larson et al., 1986; Patil et al., 1998)) was then applied to the slice for $2 \mathrm{~s}$ at maximal $(100 \%)$ shock strength (tetanus). The potentiating train was not applied to control slices. The potentials were recorded for 30 min following the tetanus, with the test stimulus pulses returned to the pre-adjusted $50-60 \%$ strength level, for the establishment of a new baseline (Reference). After this step, the response to the maximal $(100 \%)$ shock strength stimulus was recorded for eight trials at $10 \mathrm{~s}$ intervals (the responses to the maximal strength pulses are not shown in the following figures for clarity). Carbachol, at a $50 \mu \mathrm{M}$ concentration, was bath applied during the next 30 min period; the test pulse was returned to the original 50-60\% strength level at $30 \mathrm{~s}$ intervals. Once again, the response was allowed to stabilize (Suppression). The $100 \%$ shock strength stimulus was repeated for 10 trials (not shown). The washout period commenced at this point, and the response to the test pulse was recorded for another $30 \mathrm{~min}$ at the end of which we recorded the washout potentials (wash). The 100\% stimulus was applied once more at the end of the experiment to ensure the viability of the slice (not shown). In control experiments, the same procedure was followed but the potentiating train was omitted. The entire experimental protocol required approximately one hour and forty-five minutes to complete.

effect of bath application of $\mathrm{CCh}$ after the potentiating stimulation was compared to the effect of CCh in slices in which the potentiating stimulus had not been delivered (Fig. 2C). In a total of 12 experimental slices, a mean potentiation to $127.7 \%( \pm 5.9)$ of the baseline was obtained (LTP reference compared to Baseline in Figs. $3 \mathrm{~A}$ and $\mathrm{C}$ ). In the control slices, the potentiating train was omitted and the unpotentiated reference potential was compared to baseline (Control reference compared to Baseline in Figs. 3B and C). After the potentiating stimulus, $50 \mu \mathrm{M}$ CCh was washed into the bath and the stabilized potentials were recorded after $30 \mathrm{~min}$ of $\mathrm{CCh}$ in both experimental (LTP suppression compared to Baseline in Figs. 3A and C) and control slices (Control suppression compared to Baseline in Figs. 3B and C). The average slopes of the suppressed potentials under carbachol went to $81.9 \%( \pm 5.9)$ of the baseline recorded at the beginning of the experiment in experimental slices and to $80.2 \%( \pm 5.2)$ of the baseline in control slices (LTP suppression and Control suppression compared to Baseline in Fig. 3A-C). There was no significant difference between the experimental and control slices when the $\mathrm{CCh}$ potentials were compared to the baseline. This suggests that the newly potentiated strength of the synapses was fully suppressed by carbachol, whereas the initial strength was equally suppressed in both conditions. This is consistent with our modeling results suggesting that $\mathrm{CCh}$ should suppress previously 
A

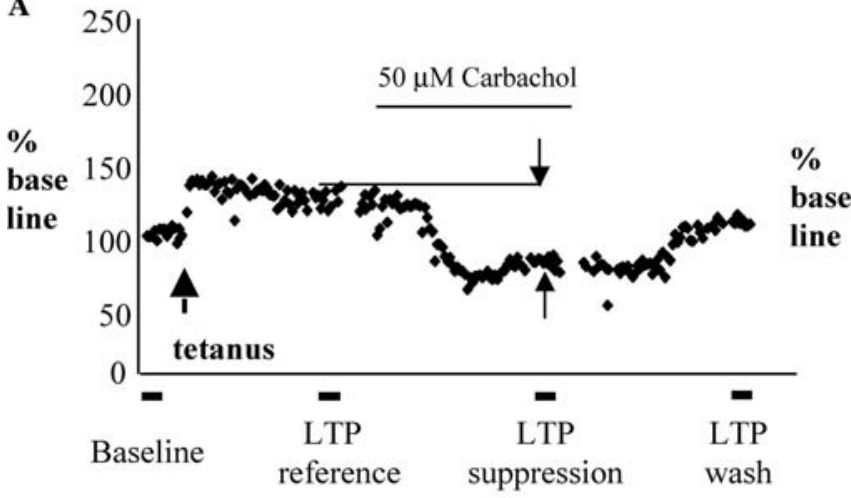

30 minutes

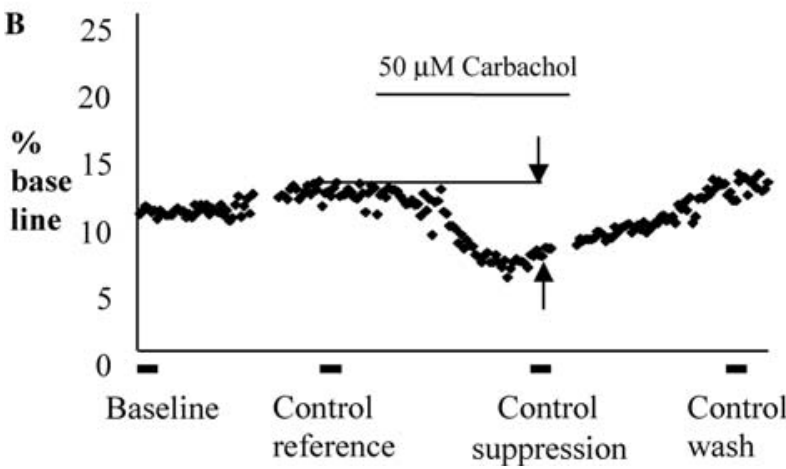

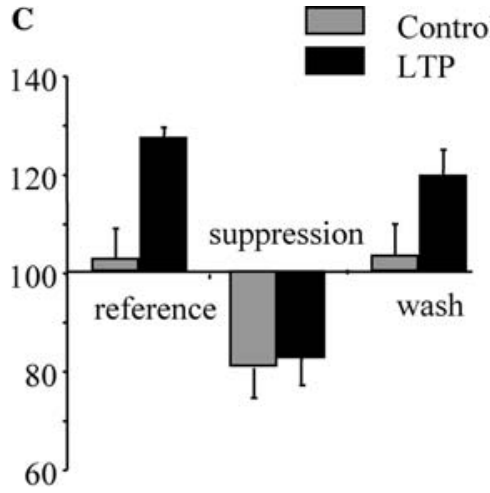

D $\quad \%$ suppression

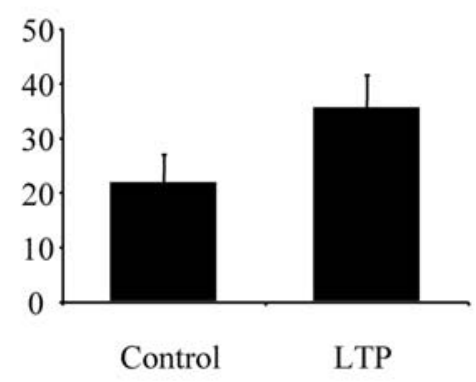

Fig. 3. Results from electrophysiological experiments. (A) Time course of a sample experiment. Each data point corresponds to the slope obtained from a single stimulation; all data points are expressed as percentage of the slopes of the baseline potentials. The baseline was obtained as the average slope of potentials resulting from 5 subsequent test pulses obtained at $30 \mathrm{~s}$ intervals during the first five minutes of the experiment (Baseline), after which the potentiating pulse train was applied (tetanus). Thirty minutes after the delivery of the tetanus, the reference potentials for each LTP experiment were recorded as the average of 5 potentials recorded at $30 \mathrm{~s}$ intervals ( $L T P$ reference). Subsequently, $50 \mu \mathrm{M}$ carbachol was washed into the bath and the slope of the suppressed potentials was calculated by averaging the responses to five potentials after 30 min (LTP suppressiond). The $\mathrm{CCh}$ was then washed out for $30 \mathrm{~min}$ and the average potential after washout was recorded (LTP wash). (B) Sample control experiment. The control experiments followed the same protocol as described in (B), except for the absence of the potentiating stimulus train. (C) Average values of slopes of recorded potentials over the time course of all experimental and control slices. The slopes of the individual synaptic potentials were automatically calculated using existing software. Each data point was calculated from the average of 5 potentials obtained at 30 s intervals and sample means and standard errors were computed for each condition. Paired $t$ tests were used to compare the means of the slopes obtained within each experimental group before normalization. The graph shows the means and standard errors of the slopes of the reference potentials (as a percentage of baseline) recorded $30 \mathrm{~min}$ after the application of the tetanus (or $30 \mathrm{~min}$ after the recording of the baseline for the control experiments) (reference), the slopes of the suppressed potentials $30 \mathrm{~min}$ after the bath application of $50 \mu \mathrm{M} \mathrm{CCh}$ (suppression), and the slopes of the potentials recorded $30 \mathrm{~min}$ after the beginning of the wash (wash) for both the experimental $(L T P)$ and control experiments (Control). (D) Suppression of potentials in $50 \mu \mathrm{M} C C h$. The suppression value was calculated as the ratio between the slopes of the suppressed potentials and the reference potentials (LTP suppression/LTP reference and Control suppression/control reference). The suppression values obtained in the two conditions were compared using single factor analysis of variance and significance values of $p<.05$ were accepted. The graph shows the amount of suppression as compared to the reference for control experiments (Control: 100.0*(1.0 - [Control suppression]/[Control reference])) and LTP experiments (LTP: 100.0*(1.0 - [LTP suppression]/[LTP reference])).

strengthened fibers more strongly than unpotentiated fibers. Both in LTP slices and in control slices the potentials fully recovered to the amplitude of the reference potentials (Wash in Fig. 3C) after 30 min of washout. As a further test of the hypothesis from the model that $\mathrm{CCh}$ should suppress previously strengthened fibers more strongly than unpotentiated fibers, we also compared the suppressed potentials under $\mathrm{CCh}$ to the reference potentials obtained after the application of the potentiating stimulus. In experimental slices, the ratio between the slopes of the suppressed potentials and those of the reference potentials after potentiation was $64.38 \%$ ( \pm 3.9$)$ yielding an average suppression of $35.62 \%$ (LTP suppression vs. LTP reference). In control slices, the average ratio was $78.1( \pm 5.1)$ yielding an average suppression of $21.9 \%$ (Control suppression vs. control reference). There was a statistically significant difference between the two experimental conditions $(d f=1$; $F=10.75 ; p<.002$ by single factor analysis of variance).

Our experimental data clearly support the theoretical requirement that cholinergic receptor activation should 
more strongly suppress newly potentiated synapses than the synapses contributing to baseline responses. The cholinergic suppression we observed in "naïve" slices was significantly lower than that observed in potentiated slices. One possible interpretation of the data is that in both cases, the initial unpotentiated potentials recorded at baseline are suppressed by approximately $20 \%$, whereas the additional, potentiated component of the potentials in the experimental slices is totally suppressed. Another interpretation is that in the experi- mental slices, both components of the potential are suppressed by the same amount, i.e. approximately $40 \%$ (see Eq. (1) and Fig. 4).

\subsection{Computational modeling results using the parameters gathered in the brain slice experiment}

As noted above, the experimental data can be interpreted in two ways: (1) Stronger suppression of the newly added efficacy of synapses coupled with weaker

A

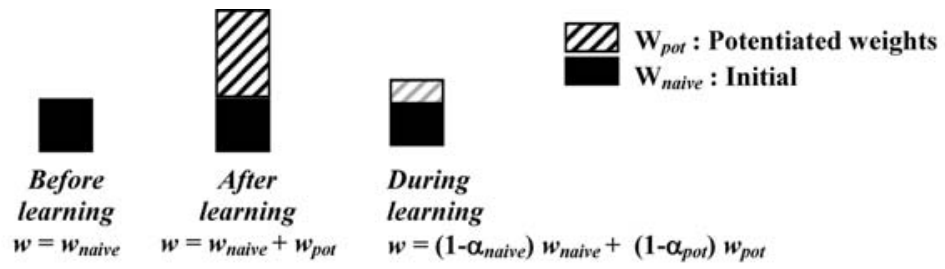

B
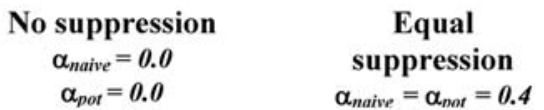
Selective suppression
$\alpha_{\text {naive }}=0.25$
$\alpha_{\text {pot }}=1.0$

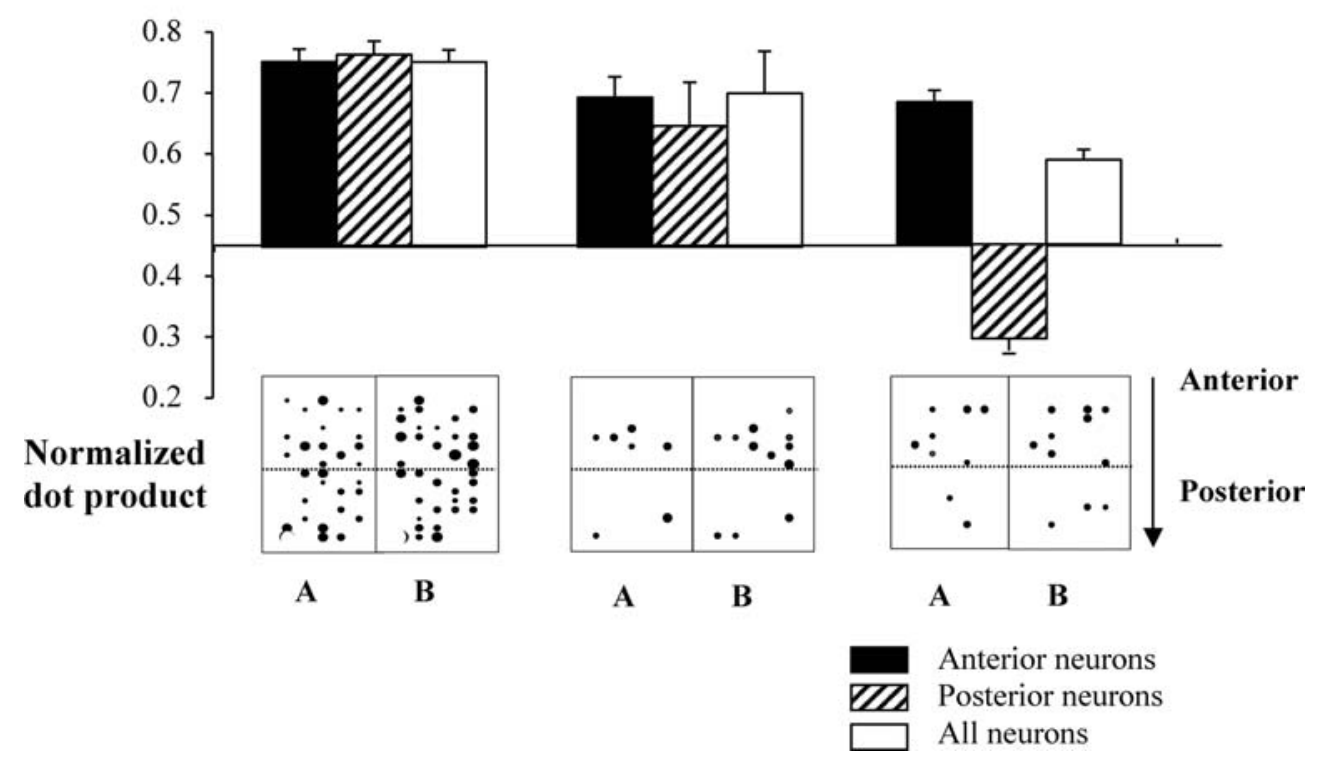

Fig. 4. Simulation results. (A) Implementation of experimental results in the simulations. When a network is constructed, all intrinsic association fibers have an initial connection strength $w=w_{\text {naïve }}$ chosen from a uniform random distribution. After the learning of an input pattern, some connections will have been strengthened and a potentiated part $w_{\text {pot }}$ of the total connection strength is added to the initial strength. During learning phases, cholinergic suppression acts on the initial connection strength $w_{\text {naive }}$ with a suppression factor $\alpha_{\text {naïve }}$ and on the potentiated connection strength $w_{\text {pot }}$ with a suppression factor $\alpha_{\text {pot }}$. B. Average overlap between the neural responses to two random input patterns after learning under different conditions. The bar-graph represents the averages and standard errors calculated from 25 simulations with different initial conditions and different random input patterns, as well as one example for each combination of parameters. Each of the two input patterns activated $20 \%$ of the anterior neurons and the two patterns overlapped by $50 \%$. To obtain the average overlap between the neural responses to the two input patterns after learning, we calculated the normalized dot product between the neural activities evoked by each pattern during $120 \mathrm{~ms}$. We first computed which neurons were responding with increased firing (as compared to baseline) during the application of the stimulus. We then calculated the normalized dot product between the two sets of active neurons in response to each of the two stimuli. The graph shows the dot products between anterior responses (Anterior neurons), posterior responses (Posterior neurons) and the responses of all neurons in the network (All neurons) after three different learning conditions. No suppression: neither the naïve nor the potentiated components of the synaptic weights were suppressed during learning $\left(\alpha_{\text {naive }}=\alpha_{\text {pot }}=0.0\right)$. Equal suppression: the naïve and the potentiated components of the synaptic weights were suppressed by the same amount $\left(\alpha_{\text {naive }}=\alpha_{\text {pot }}=0.4\right)$. Selective suppression: the naïve components of the synaptic weights were substantially less suppressed than the potentiated components $\left(\alpha_{\text {naïve }}=0.2 ; \alpha_{\text {pot }}=1.0\right)$. Note the much lower dot product between posterior responses in this condition. The frames below the bar graph show the average activities in the network in response to two learned input patterns (A and B) after learning with the appropriate combination of $\alpha_{\text {naïve }}$ and $\alpha_{\text {pot. }}$. The average activities (number of action potentials during the application of the stimulus) of all neurons in the simulations are represented by the size of the dots. 
suppression of the original component of efficacy or (2) Equal suppression of both potentiated strength and baseline strength. Our computational model allowed testing of the functional implications of these two possibilities. We have tested the effect of including the suppression parameters calculated from the experimental results on the properties of the activity patterns arising in our computational model (Fig. 4A). For each connection, we separated the initial connection strength $\left(w_{\text {naïve }}\right)$ from the potentiated part of the connection weight $\left(w_{\text {pot }}\right)$. During the learning process, we could then separately suppress each component of the synaptic weight by a given factor $\left(\alpha_{\text {naive }}\right.$ and $\left.\alpha_{\text {pot }}\right)$. Since the effect of cholinergic suppression of association fibers on memory capacity has been extensively studied previously, we focussed our attention on the self-organizing properties of the network: we analyzed the effect of different levels of suppression during the learning process on the overlap between the neural representations in response to learned input patterns after the learning process. The parameters $\alpha_{\text {naive }}$ and $\alpha_{\text {pot }}$ were varied systematically over a wide range of values, and total posterior cortical activity and percent overlap were analyzed. Here we focus on a subset of parameters illustrating the computational relevance of the experimental data. When no suppression of intrinsic fibers was present during learning $\left(\alpha_{\text {naïve }}=\alpha_{\text {pot }}=0.0\right)$, high overlap between the two input patterns led to high interference in the anterior portion of the network, which in turn recruited large portions of highly overlapping neurons in the posterior portion of the network (Fig. 4B, No suppression). If both naïve and potentiated components of the synaptic weights were suppressed equally during the learning process $\left(\alpha_{\text {naive }}=\alpha_{\text {pot }}=0.4\right)$, no or very few posterior neurons were recruited and the overlap between the neural response patterns was mainly determined by the activity of the anterior neurons (Fig. 4B, Equal suppression). In contrast, when the naïve component of the synaptic weights was only slightly suppressed $\left(\alpha_{\text {naive }}=0.2\right)$ and the potentiated component was completely suppressed $\left(\alpha_{\mathrm{pot}}=1.0\right)$, as suggested by the data, only a few non-overlapping neurons in the posterior part of the network were recruited by each pattern and the overlap was diminished by the contribution of posterior neurons (Fig. 4B, Selective suppression). The overlap between the self-organized representations in the posterior network was much lower than that in the anterior network and thus significantly diminished the total overlap. Thus, selective suppression allows formation of more distinct representations of odors, which could enhance discrimination behavior.

\section{Discussion}

The computational model presented here demonstrates that the self-organization of odor responses in the posterior piriform cortex functions best when cholinergic modulation suppresses excitatory glutamatergic synaptic transmission more strongly at previously modified synapses. The experimental data presented here supports this result by demonstrating that the cholinergic agonist carbachol (CCh) more strongly suppresses potentiated synapses, while having a weaker effect on baseline synaptic strength. Finally, further modeling shows that self-organization works best if this suppression is selective for the synaptic strength added by potentiation, rather than the initial strength of synaptic transmission. These results demonstrate the potential role of cholinergic modulation of synaptic transmission in the brain mechanisms regulating formation of distinct responses to similar sensory stimuli.

Most implementations of abstract networks performing associative memory tasks employ the strategy of ignoring the calculated changes in synaptic strength during the learning process (Ambros-Ingerson et al., 1990; Amit, Evans, \& Abeles, 1990; Hopfield, 1982), but relatively few experiments have addressed this strategy. The experimental data presented here suggests that acetylcholine could allow this property. Models of self-organization have also shown that the activity induced by previously modified connections must be regulated to allow stable learning and prevent interference between distinct representations (AmbrosIngerson et al., 1990; Carpenter \& Grossberg, 1993). We here propose that while suppression of excitatory synaptic transmission during the storage process is necessary for stable network activity, this suppression should be limited to previously potentiated synapses, which are already part of the representation of a stored pattern. In that case, the very low suppression of unpotentiated synapses allows the recruitment of additional context neurons while the higher suppression of the previously potentiated synpases ensures that any given synapse has a low probability to be recruited by two subsequent patterns. Our modeling hypothesis is strongly supported by our experimental data, which shows a significantly higher suppression of previously strengthened synapses as compared to naïve synapses by the cholinergic agonist $\mathrm{CCh}$. While functionally similar suppressions of synaptic changes during the learning process have been employed by most models of associative memory, we now have provided experimental support for this theoretical construct. Note that cholinergic modulation is much stronger in layer Ib connections between anterior and posterior piriform cortex than in layer Ia connections from the olfactory bulb. However, there is some suppression of transmission in layer Ia, and this suppression should also be selective for strengthened synapses in order to maintain effective self-organization. 


\subsection{Relation to other data on the olfactory system}

Models demonstrate that the loss of this modulatory effect of acetylcholine would cause a greater overlap in the neural representations formed in response to odors. A greater overlap in neuronal responses would be consistent with the behavioral data showing that scopolamine causes enhanced interference between overlapping odor pairs (DeRosa \& Hasselmo, 2000) and prevents the enhanced acuity caused by odor conditioning (Fletcher \& Wilson, 2002). These effects could also contribute to the decreased behavioral discrimination of similar odorants or odor pairs caused by selective lesions of the cholinergic neurons in the horizontal limb of the diagonal band of Broca, which provides cholinergic innervation of the olfactory cortex and olfactory bulb (DeRosa, Hasselmo, \& Baxter, 2001; Linster et al., 2001). The decreased neural separation of odors could also contribute to the effect of cholinergic blockade on odorant short-term memory (Ravel et al., 1994) and odor-reward associations (Roman, Simonetto, \& Soumireu-Mourat, 1993).

These models are consistent with electrophysiological data on neuronal responses of olfactory cortex in vivo (Wilson, 1998, 2001, 2003). Recordings by Wilson have shown that piriform cortex neurons show strong habituation to repeated presentations of odors (Wilson, 1998). In this paradigm, scopolamine causes enhanced cross-habituation of odor responses in individual neuronal responses (Wilson, 2001), possibly due to an increased overlap in the neural representation of odors such as that appearing in Figs. 1C or 4B ("no suppression"). Piriform cortex neurons also show an increase in discrimination of odorant mixtures from their component parts, which does not appear in olfactory bulb (Wilson, 2003). Though the recordings were done in anterior piriform cortex, the discrimination response could result from enhanced formation of non-overlapping representations in posterior piriform cortex (as in Figs. 1C, 4B-"Selective suppression"), which then influence anterior piriform cortex activity.

\subsection{Relation to other physiological data}

Many studies have demonstrated that cholinergic modulation enhances long-term potentiation in cortical structures including hippocampus, neocortex and piriform cortex (Blitzer et al., 1990; Brocher et al., 1992; Hasselmo \& Barkai, 1995; Huerta \& Lisman, 1993; Patil et al., 1998). However, the experiment presented here addresses a separate issue. Rather than infusing acetylcholine before induction of long-term potentiation, here we induce long-term potentiation and then observe the magnitude of suppression after long-term potentiation. In this manner, we were able to show stronger cholinergic presynaptic inhibition of potentiated synapses.
Previous studies on the cholinergic enhancement of long-term potentiation demonstrated cholinergic suppression of synaptic transmission, but did not quantify the magnitude of this suppression after induction of LTP.

The work presented here has an important relationship to recently published data showing stronger cholinergic suppression of "functional" synapses compared to "silent" synapses (de Sevilla, Cabezas, de Prada, Sanchez-Jimenez, \& Buno, 2002). In that study, "functional" synapses refers to synapses which contain nonNMDA receptors, allowing clear appearance of EPSPs in response to minimal stimulation delivered at neuronal resting potential. These synapses showed 36\% suppression by carbachol. In contrast, "silent" synapses are synapses containing predominantly NMDA receptors, which can be observed best when the postsynaptic cell is depolarized to allow synaptic current through NMDA receptors in response to minimal stimulation. These "silent" synapses showed only 7\% suppression by carbachol and muscarine. Note that the use of minimal stimulation (de Sevilla et al., 2002) prevents NMDA currents from appearing in neurons at resting potential, whereas the stronger stimulation used in our study probably caused more extensive activation of NMDA currents due to strong depolarization caused by extensive AMPA currents on the dendrites. Thus, synapses which are "silent" with minimal stimulation (de Sevilla et al., 2002) can probably contribute significantly to the field potentials evoked with stronger stimulation in our study. Those recent results provide a potential mechanism for the effects we have observed, because long-term potentiation has been shown to involve increased insertion of AMPA receptors into the postsynaptic membrane. In this manner, the newly potentiated synaptic strength could be more sensitive to cholinergic modulation of "functional" synapses, whereas the baseline activity could involve a less sensitive set of synapses. The evidence for selective effects of carbachol at one type of glutamatergic synapse but not another in that previous study (de Sevilla et al., 2002) supports the separate modulatory influence reported here.

\subsection{Relationship to cholinergic modulation of self-orga- nization in other systems}

In other systems, acetylcholine has been shown to modulate the response to sensory stimuli. In the olfactory bulb, acetylcholine appears to play an important role in increasing the discriminability of related odor stimuli (Linster \& Cleland, 2002; Linster et al., 2001). In the auditory cortex, acetylcholine has been shown to play a role in changes in the pattern of response to different frequencies when specific frequencies are associated with shock (Weinberger, 1998). In fact, the pattern of physiological response to auditory frequencies 
can be directly altered by pairing cholinergic modulation with presentation of a specific tone (Metherate \& Weinberger, 1990), and this pairing can induce changes on a behavioral level (McLin, Miasknikov, \& Weinberger, 2002). Similarly, acetylcholine appears to regulate changes in the pattern of cutaneous response properties in somatosensory cortex associated with learning (Dykes, 1997). These studies show that acetylcholine regulates the properties of self-organization. The initial formation of frequency sensitivity in auditory cortex and somatopic organization in somatosensory cortex both probably involve self-organization mechanisms similar to those used in modeling primary visual cortex (Erwin, Obermayer, \& Schulten, 1995). In the training paradigms of Weinberger or Dykes, acetylcholine appears to regulate this self-organization by enhancing plasticity during presentation of specific sensory stimuli, allowing those specific sensory stimuli to cause a stronger or more prominent neuronal response.

The self-organization described here differs somewhat from the traditional self-organization models of primary visual cortex. Models of primary visual cortex usually focus on modification of the afferent input fibers to form feature detectors and topographic maps (Erwin et al., 1995; Von der Malsburg, 1973). In contrast, in the model presented here, we focus on self-organization of intrinsic connections within the piriform cortex (recurrent connections and connections from anterior to posterior cortex). This self-organization is similar in principle, but differs in its anatomical focus on intrinsic rather than afferent connections. This focus on self-organization of intrinsic connections has been used in models of hippocampus (Levy, 1996; Wallenstein \& Hasselmo, 1997; Wu et al., 1996) and piriform cortex (Linster et al., 1996). However, given that most excitatory connections even in primary visual cortex are intrinsic, it is likely that this type of self-organization is very important for regions such as primary visual cortex. The suppression of excitatory recurrent connectivity by cholinergic receptors has been shown in primary visual cortex (Brocher et al., 1992; Kimura, Fukuda, \& Tsumoto, 1999). Cholinergic modulation has been shown to enhance the selectivity of visual cortical neurons (Murphy \& Sillito, 1991; Sillito \& Kemp, 1983). This could result from cholinergic depolarization of neurons causing enhanced response to input, while the suppression of excitatory transmission would maintain or enhance the direction selectivity of neurons (Murphy \& Sillito, 1991; Sillito \& Kemp, 1983). This could also play a vital role in allowing self-organization without an explosion of excitatory activity. The changes in response properties observed with cholinergic modulation in other sensory cortices could also involve selective recruitment of additional neurons via intrinsic connections, which would benefit from the principles described here.

The evidence from physiological and behavioral experiments, and the integration of this work with com- putational modeling suggests an important role for acetylcholine in enhancing the selective response to sensory input and the encoding of new representations. This appears important both for the modification of sensory response properties (Dykes, 1997; Linster et al., 2001; Weinberger, 1998) and for preventing interference during encoding of episodic memories (Atri et al., 2003; Ghonheim \& Mewaldt, 1977; Hasselmo et al., 1995). On a global level, these modulatory effects of acetylcholine may even regulate the relative role of different structures during different phases of behavior. Research has shown that levels of acetylcholine in the hippocampus may mediate its initial importance for encoding of spatial position information for performing a plus maze task (Chang \& Gold, 2003) whereas the later shift to a turning response strategy could involve increases in acetylcholine in the striatum. The levels of acetylcholine in hippocampus vs. striatum even correlate with individual differences in response strategy (McIntyre, Marriott, \& Gold, 2003), suggesting a powerful role of acetylcholine in regulating the functional role of different regions.

In summary, changes in acetylcholine levels regulate the role of different regions in plasticity and behavior by setting encoding dynamics due to cellular effects of acetylcholine. The modeling and physiology results presented here demonstrate how selective cholinergic suppression of modified synapses can enhance the ability of cortical structures to form new representations of sensory stimuli.

\section{Acknowledgments}

Supported by NIMH R01 grants MH61492, MH60013 and Conte Center grant MH60450.

\section{References}

Aigner, T. G., \& Mishkin, M. (1986). The effects of physostigmine and scopolamine on recognition memory in monkeys. Behavioral Neuroscience, 45, 81-87.

Ambros-Ingerson, J., Granger, R., \& Lynch, G. (1990). Simulation of paleocortex performs hierarchical clustering. Science (Washington DC), 247(4948).

Amit, D. J., Evans, M. R., \& Abeles, M. (1990). Attractor neural networks with biological probe records. Network, 1, 381-405.

Anderson, J. A. (1972). A simple neural network generating an interactive memory. Mathematical Biosciences, 14, 197-220.

Atri, A., Sherman, S. J., Norman, K. A., Kirchhoff, B. A., Nicolas, M. M., Greicius, M. D., Cramer, S. C., Breiter, H. C., Hasselmo, M. E., \& Stern, C. E. (2003). Blockade of central cholinergic receptors impairs new learning and increases proactive interference in a word paired-associate memory task. Behavioral Neuroscience, in press.

Barkai, E., \& Hasselmo, M. E. (1994). Modulation of the input/output function of rat piriform cortex pyramidal cells. Journal of Neurophysiology, 72, 644-658.

Barkai, E., Bergman, R. E., Horwitz, G., \& Hasselmo, M. E. (1994). Modulation of associative memory function in a biophysical 
simulation of rat piriform cortex. Journal of Neurophysiology, 72(2), 659-677.

Benardo, L. S., \& Prince, D. A. (1982). Ionic mechanisms of cholinergic excitation in mammalian hippocampal pyramidal cells. Brain Research, 249, 333-344.

Blitzer, R. D., Gil, O., \& Landau, E. M. (1990). Cholinergic stimulation enhances long-term potentiation in the CA1 region of rat hippocampus. Neuroscience Letters, 119, 207-210.

Brocher, S., Artola, A., \& Singer, W. (1992). Agonists of cholinergic and noradrenergic receptors facilitate synergistically the induction of long-term potentiation in slices of rat visual cortex. Brain Research, 573, 27-36.

Carpenter, G. A., \& Grossberg, S. (1993). Normal and amnesic learning, recognition and memory by a neural model of cortico-hippocampal interactions. Trends in Neurosciences, 16, 131-137.

Chang, Q., \& Gold, P. E. (2003). Switching memory systems during learning: Changes in patterns of brain acetylcholine release in the hippocampus and striatum in rats. Journal of Neuroscience, 23(7), 3001-3005

DeRosa, E., \& Hasselmo, M. E. (2000). Muscarinic cholinergic neuromodulation reduces proactive interference between stored odor memories during associative learning in rats. Behavioral Neuroscience, 114, 32-41.

DeRosa, E., Hasselmo, M. E., \& Baxter, M. G. (2001). Contribution of the cholinergic basal forebrain to proactive interference from stored odor memories during associative learning in rats. Behavioral Neuroscience, 115(2), 314-327.

de Sevilla, D. F., Cabezas, C., de Prada, A. N., Sanchez-Jimenez, A., \& Buno, W. (2002). Selective muscarinic regulation of functional glutamatergic Schaffer collateral synapses in rat CA1 pyramidal neurons. Journal of Physiology, 545, 51-63.

Dutar, P., \& Nicoll, R. A. (1988). Classification of muscarinic responses in hippocampus in terms of receptor subtypes and 2ndmessenger systems - electrophysiological studies in vitro. Journal of Neuroscience, 8, 4214-4224.

Dykes, R. W. (1997). Mechanisms controlling neuronal plasticity in somatosensory cortex. 75(5), 535-545.

Erwin, E., Obermayer, K., \& Schulten, K. (1995). Models of orientation and ocular dominance columns in the visual cortex: a critical comparison. Neural Computation, 7(3), 425-468.

Fletcher, M. L., \& Wilson, D. A. (2002). Experience modifies olfactory acuity: Acetylcholine-dependent learning decreases behavioral generalization between similar odorants. Journal of Neuroscience, 22 (RC201), 1-5.

Ghonheim, M. M., \& Mewaldt, S. P. (1977). Studies on human memory: The interactions of diazepam, scopolamine and physostigmine. Psychopharmacology, 52, 1-6.

Gil, Z., Connors, B. W., \& Amitai, Y. (1997). Differential regulation of neocortical synapses by neuromodulators and activity. Neuron, 19 , $679-686$.

Grossberg, S. (1976). Adaptive pattern classification and universal recoding: II. Feedback, expectation, olfaction, illusions. Biological Cybernetics, 23(4), 187-202.

Haberly, L. B. (1985). Neuronal circuitry in olfactory cortex: anatomy and functional implications. Chemical Senses, 10, 219-238.

Haberly, L. B., \& Bower, J. M. (1989). Olfactory cortex model circuit for study of associative memory. Trends in Neurosciences, 12(7).

Haberly, L. B., \& Price, J. L. (1977). The axonal projection patterns of the mitral and tufted cells of the olfactory bulb in the rat. Brain Research, 129, 152-157.

Hasselmo, M. E. (1995). Neuromodulation and cortical function: modeling the physiological basis of behavior. Behavioral Brain Research, 67(1), 1-27.

Hasselmo, M. E., Anderson, B. P., \& Bower, J. M. (1992). Cholinergic modulation of cortical associative memory function. Journal of Neurophysiology, 67(5), 1230-1246.
Hasselmo, M. E., \& Barkai, E. (1995). Cholinergic modulation of activity-dependent synaptic plasticity in rat piriform cortex. Journal of Neuroscience, 15, 6592-6604.

Hasselmo, M. E., \& Bower, J. M. (1992). Cholinergic suppression specific to intrinsic not afferent fiber synapses in rat piriform olfactory cortex. Journal of Neurophysiology (Bethesda), 67(5).

Hasselmo, M. E., \& Bower, J. M. (1993). Acetylcholine and memory. Trends in Neuroscience, 16, 218-222.

Hasselmo, M. E., Linster, C., Patil, M., Ma, D., \& Cekic, M. (1997). Noradrenergic suppression of synaptic transmission may influence cortical signal-to-noise ratio. Journal of Neurophysiology, 77(6), 3326-3339.

Hasselmo, M. E., \& McGaughy, J. (2003). High acetylcholine levels set circuit dynamics for attention and encoding and low acetylcholine levels set dynamics for consolidation. Progress in Brain Research, $145,207-231$.

Hasselmo, M. E., Schnell, E., \& Barkai, E. (1995). Dynamics of learning and recall at excitatory recurrent synapses and cholinergic modulation in rat hippocampal region CA3. Journal of Neuroscience, 15(27 Pt), 5249-5262.

Hasselmo, M. E., \& Wyble, B. P. (1997). Free recall and recognition in a network model of the hippocampus: simulating effects of scopolamine on human memory function. Behavioral Brain Research, 89(1-2), 1-34.

Hopfield, J. J. (1982). Neural networks and physical systems with emergent selective computational abilities. Proceedings of the National Academy of Sciences of the United States of America, 79, 2554-2559.

Hounsgaard, J. (1978). Presynaptic inhibitory action of acetylcholine in area CA1 of the hippocampus. Experimental Neurology, 62, 787797.

Hsieh, C. Y., Cruikshank, S. J., \& Metherate, R. (2000). Differential modulation of auditory thalamocortical and intracortical synaptic transmission by cholinergic agonist. Brain Research, 880, 51-64.

Huerta, P. T., \& Lisman, J. E. (1993). Heightened synaptic plasticity of hippocampal CA1 neurons during a cholinergically induced rhythmic state. Nature, 364, 723-725.

Hunter, A. J., \& Murray, T. K. (1989). Cholinergic mechanisms in a simple test of olfactory learning in the rat. Psychopharmacology, 99, 270-275.

Jung, M. W., Larson, J., \& Lynch, G. (1990). Long-term potentiation of monosynaptic EPSPs in rat piriform cortex in vitro. Synapse, 6 , 279-283.

Kanter, E. D., \& Haberly, L. B. (1990). NMDA-dependent induction of long-term potentiation in afferent and association fiber systems of piriform cortex in vitro. Brain Research, 525, 175-179.

Kanter, E. D., \& Haberly, L. B. (1993). Associative long-term potentiation in piriform cortex slices requires Gaba-A blockade. Journal of Neuroscience, 13(6), 2477-2482.

Kimura, F., Fukuda, M., \& Tsumoto, T. (1999). Acetylcholine suppresses the spread of excitation in the visual cortex revealed by optical recording: possible differential effect depending on the source of input. European Journal of Neuroscience, 11(10), 35973609.

Kohonen, T. (1984). Correlation matrix memories. IEEE Transactions on Computers, C-21, 353-359.

Levy, W. B. (1996). A sequence predicting CA3 is a flexible associator that learns and uses context to solve hippocampal-like tasks. Hippocampus, 6(6), 579-590.

Linster, C., \& Cleland, T. A. (2002). Cholinergic modulation of sensory representations in the olfactory bulb. Neural Networks, 15, 709-717.

Linster, C., Hasselmo, M. F., \& Gervais, R. (1995). Interactions between olfactory bulb and olfactory cortex in a neural model of odor processing. In 25th Annual meeting of the society for neuroscience, San Diego, California,USA, November 11-16, 1995 (Vol. 21, p. 1747). Society for Neuroscience Abstracts. 
Linster, C., Wyble, B. P., \& Hasselmo, M. E. (1999). Electrical stimulation of the horizontal limb of the diagonal band of Broca modulates population EPSPs in piriform cortex. Journal of Neurophysiology, 81(6), 2737-2742.

Linster, C., Garcia, P. A., Hasselmo, M. E., \& Baxter, M. G. (2001). Selective loss of cholinergic neurons projecting to the olfactory system increases perceptual generalization between similar, but not dissimilar, odorants. Behavioral Neuroscience, 115, 826-833.

Madison, D. V., \& Nicoll, R. A. (1984). Control of the repetitive discharge of rat CA1 pyramidal neurones in vitro. Journal of Physiology, 354, 319-331.

Marr, D. (1971). Simple memory: a theory for archicortex. Philosophical transactions of the Royal Society of London B, 262, 23-81.

McGaughy, J., \& Sarter, M. (1998). Sustained attention performance in rats with intracortical infusions of $192 \mathrm{IgG}$-saporin-induced cortical cholinergic deafferentation: effects of physostigmine and FG 7142. Behavioral Neuroscience, 110, 247-265.

McGaughy, J., Kaiser, T., \& Sarter, M. (1996). Behavioral vigilance following infusions of $192 \mathrm{IgG}$-saporin into the basal forebrain: selectivity of the behavioral impairment and relation to cortical AChE-positive fiber density. Behavioral Neuroscience, 110, 247265.

McIntyre, C. K., Marriott, L. K., \& Gold, P. E. (2003). Patterns of brain acetylcholine release predict individual differences in preferred learning strategies in rats. Neurobiology of Learning and Memory, 79(2), 177-183.

McLin, D. E., III, Miasknikov, A. A., \& Weinberger, N. M. (2002). Induction of behavioral associative memory by stimulation of the nucleus basalis. Proceedings of the National Academy of Sciences of the United States of America, 99(6), 4002-4007.

McNaughton, B., \& Morris, R. (1987). Hippocampal synaptic enhancement and memory storage within a distributed memory system. Trends in Neuroscience, 10, 408-415.

Murphy, P. C., \& Sillito, A. M. (1991). Cholinergic enhancement of direction selectivity in the visual cortex of the cat. Neuroscience, 40(1), 13-20.

Patil, M. M., \& Hasselmo, M. E. (1999). Modulation of inhibitory synaptic potentials in the piriform cortex. Journal of Neurophysiology, 81(5), 2103-2118.

Patil, M. M., Linster, C., Lubenov, E., \& Hasselmo, M. E. (1998). Cholinergic agonist carbachol enables associative long-term potentiation in piriform cortex slices. Journal of Neurophysiology, 80(5), 2467-2474.
Ravel, N., Elaagouby, A., \& Gervais, R. (1994). Scopolamine injection into the olfactory bulb impairs short-term olfactory memory in rats. Behavioral Neuroscience, 108, 317-324.

Roman, F. S., Simonetto, I., \& Soumireu-Mourat, B. (1993). Learning and memory of odor-reward association: selective impairment following horizontal diagonal band lesions. Behavioral Neuroscience, 107, 72-81.

Sherman, S. J., Atri, A., Hasselmo, M. E., Stern, C. E., \& Howard, M. W. (2003). Scopolamine impairs human recognition memory: Data and modeling. Behavioral Neuroscience, 117(3), 526-539.

Sillito, A. M., \& Kemp, J. A. (1983). Cholinergic modulation of the functional organization of the cat visual cortex. Brain Research, 289, 143-155.

Von der Malsburg, C. (1973). Self-organization of orientation sensitive cells in the striate cortex. Kybernetik, 14(2), 85-100.

Wallenstein, G. V., \& Hasselmo, M. E. (1997). GABAergic modulation of hippocampal population activity: sequence learning, place field development, and the phase precession effect. Journal of Neurophysiology, 78(1), 393-408.

Weinberger, N. M. (1998). Physiological memory in primary auditory cortex: Characteristics and mechanisms. Neurobiology of Learning and Memory, 70, 226-251.

Williams, S. H., \& Constanti, A. (1988). Quantitative effects of some muscarinic agonists on evoked surface-negative field potentials recorded from the guinea-pig olfactory cortex slice. British Journal of Pharmacology, 93, 846-854.

Wilson, D. A. (1998). Habituation of odor responses in the rat anterior piriform cortex. Journal of Neurophysiology, 79, 1425-1440.

Wilson, D. A. (2001). Scopolamine enhances generalization between odor representations in rat olfactory cortex. Learning \& Memory, 8 , 279-285.

Wilson, D. A. (2003). Rapid, experience induced enhancement in odorant discrimination by anterior piriform cortex neurons. Journal of Neurophysiology, 90, 65-72.

Wilson, M. A., \& Bower, J. M. (Eds.). (1988). A Computer Simulation of Olfactory Cortex With Functional Implications for Storage and Retrieval of Olfactory Information. New York: AIP Press.

Wilson, M., \& Bower, J. M. (1992). Cortical oscillations and temporal interactions in a computer simulation of piriform cortex. Journal of Neurophysiology, 67, 981-995.

Wu, X., Baxter, R. A., \& Levy, W. B. (1996). Context codes and the effect of noisy learning on a simplified hippocampal CA3 model. Biological Cybernetics, 74(2), 159-165. 